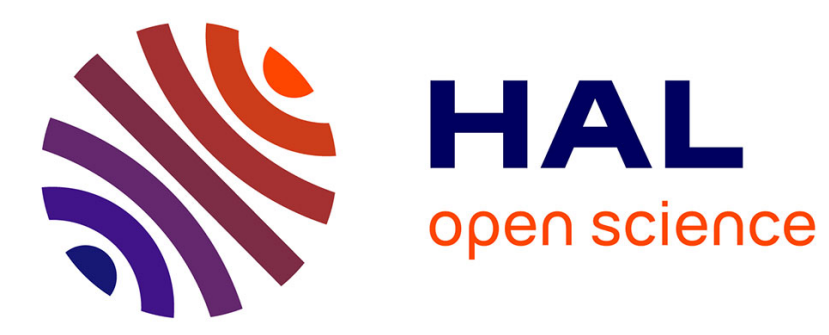

\title{
Fault Injection Resilience
}

Sylvain Guilley, Laurent Sauvage, Jean-Luc Danger, Nidhal Selmane

\section{To cite this version:}

Sylvain Guilley, Laurent Sauvage, Jean-Luc Danger, Nidhal Selmane. Fault Injection Resilience. Fault Diagnosis and Tolerance in Cryptography, Aug 2010, Santa Barbara, United States. pp.51-65, 10.1109/FDTC.2010.15 . hal-00482194v9

\section{HAL Id: hal-00482194 https://hal.science/hal-00482194v9}

Submitted on 17 Jan 2011

HAL is a multi-disciplinary open access archive for the deposit and dissemination of scientific research documents, whether they are published or not. The documents may come from teaching and research institutions in France or abroad, or from public or private research centers.
L'archive ouverte pluridisciplinaire HAL, est destinée au dépôt et à la diffusion de documents scientifiques de niveau recherche, publiés ou non, émanant des établissements d'enseignement et de recherche français ou étrangers, des laboratoires publics ou privés. 


\title{
Fault Injection Resilience
}

\author{
Sylvain GUILLEY ${ }^{1,2}$, Laurent SAUVAGE ${ }^{1,2}$, Jean-Luc DANGER ${ }^{1,2}$ and Nidhal SELMANE ${ }^{1}$ \\ ${ }^{1}$ Institut TELECOM, TELECOM ParisTech, CNRS LTCI (UMR 5141), 46 rue Barrault, 75634 Paris, France. \\ ${ }^{2}$ Secure-IC S.A.S., $37 / 39$ rue Dareau, 75014 Paris, France and 2 rue de la Châtaigneraie, 35576 Cesson Sévigné, France. \\ \{sylvain.guilley, laurent.sauvage, jean-luc.danger, nidhal.selmane\}@telecom-paristech.fr
}

\begin{abstract}
Fault injections constitute a major threat to the security of embedded systems. Errors occurring in the cryptographic algorithms have been shown to be extremely dangerous, since powerful attacks can exploit few of them to recover the full secrets. Most of the resistance techniques to perturbation attacks have relied so far on the detection of faults. We present in this paper another strategy, based on the resilience against fault attacks. The core idea is to allow an erroneous result to be outputted, but with the assurance that this faulty information conveys no information about the secrets concealed in the chip. We first underline the benefits of FIR: false positive are never raised, secrets are not erased uselessly in case of uncompromising faults injections, which increases the card lifespan if the fault is natural and not malevolent, and FIR enables a high potential of resistance even in the context of multiple faults. Then we illustrate two families of fault injection resilience (FIR) schemes suitable for symmetric encryption. The first family is a protocollevel scheme that can be formally proved resilient. The second family mobilizes a special logic-level architecture of the cryptographic module. We notably detail how a countermeasure of this later family, namely dual-rail with precharge logic style, can both protect both against active and passive attacks, thereby bringing a combined global protection of the device. The cost of this logic is evaluated as lower than detection schemes. Finally, we also give some ideas about the modalities of adjunction of FIR to some certification schemes.
\end{abstract}

Index Terms-Fault Injection Attack (FIA), symmetric block encryption, Denial of Service (DoS), Fault Injection Resilience (FIR), Differential Fault Analysis (DFA), SideChannel Attack (SCA), Dual-rail with Precharge Logic (DPL).

\section{INTRODUCTION}

Secure embedded systems such as smartcards must be tamper-resistant so as to defeat attacks that target directly their implementation. Three kinds of threats have been identified on these devices: perturbation, observation and manipulation. Perturbation attacks consist in covertly changing one data so as to either modify the chip's execution flow or force it to output incorrect results.
Observation attacks specifically target the parts of the design that manipulate secrets; their goal is to exploit unintentional side-channel leakages so as to recover sensitive information. Manipulation is an invasive attack that gives to the attacker the power of modifying the chip's functionality or of directly probing signals [37], [25].

Manipulation attacks are the most difficult to resist against, because of their intrusiveness: the device, expected to conceal data, is suddenly reduced into a whitebox system. Fortunately, manipulation attacks involve expensive laboratory equipments, trained personnel and the sacrifice of many samples during their preparation [9]. They are therefore not the most common ones. In addition, efficient countermeasures exist, such as tamper-proof modules (e.g. SISHELL and ACSIP solutions by former industrial Axalto) or active shield on top of the chip.

Observation attacks are less costly attacks, since some side-channels, such as the magnetic field, can be recorded at will without the chip even noticing it, in a non-invasive or semi-invasive manner. There also exists a wealth of counter-measures of different quality to make side-channel attacks (SCA) difficult.

Perturbation attacks require a means to alter the device's behavior, without triggering the purported countermeasures that continuously monitor the environment. Some low cost global fault injection attacks (such as overclocking [4], [23], [2], power underfeeding [74], [7], [8] or heating [28], [68], [85]) can be used against weakly protected devices. Most expensive attacks rely on a local perturbation: for instance, laser or particle shots can avoid active shields and thus manage to surgically modify data in extremely well localized zones / dates. At the opposite, those tools can also be used to cause random and extremely spread faults in space / time. With little chance, those highly multiple faults remain undetected and thus successfully alter the chip's state.

Observation attacks on cryptographic blocks usually require a couple of hundreds or thousands observations 
in absence of countermeasures. At the opposite, fault injection attacks can reveal the secret with a small number of measurements. For instance, RSA [70] computed with the Chinese Remainder Theorem (CRT) can be broken with as few as one faulty computation [14]. The last 128 bit of the key schedule of an AES [62] block cipher can be retrieved with one single well-behaved faulty encryption [84]. These exploits motivate a special focus on fault attacks. This is all the more true as theoretically sound countermeasures have been proposed for SCAs [17] but that the coverage of fault attacks is lacunar: multiple faults, either spread in space or in time, are extremely difficult to withstand with the state-of-theart countermeasures. We therefore focus on those attacks in the rest of this article.

Fault injections attacks (FIA) can basically attempt to deviate a targeted device from its nominal functionality in two ways. Either the fault can directly profit to the attacker, such as allowing her to access unauthorized pieces of information, or the fault induces a corrupted computation that the attacker post-processes to recover secrets. The first case is an attack against security mechanisms, whereas the second one targets typically the cryptographic modules. We will not cover the first case, since known methods already exist to cross-check that a punctual valid bit is indeed correct. The second case is at the heart of this paper. Indeed, checking for the correctness of all the steps of a lengthy cryptographic computation is more costly. And above all, we notice that a cryptographic system can indeed remain secure even if it outputs incorrect results. We promote in this paper the idea that, in most cryptographic protocols, it suffices to make sure the fault does not depend on any secret to maintain a provable security level. We call this protection strategy "fault injection resilience", notion abridged as "FIR".

The rest of the paper is organized as follows. The benefit of the FIR over other techniques based on detection is discussed in Sec. II. In Sec. III, some suitable techniques to implement FIR are described. A case study of a register transfer level (RTL) implementation of FIR is detailed in Sec. IV. The impact of FIR in two security certification schemes is studied in Sec. V. Finally, conclusions and perspectives are given in Sec. VI.

\section{BENEFITS OF FIR}

\section{A. State-of-the-art of Detection Mechanisms}

As already underlined, the detection of faults is traditionally the method of choice to prevent fault attacks.
In the early years of fault tolerance in secure embedded systems, analogue solutions were used. They consist in disseminating voltage, temperature, light sensors or any miscellaneous combination thereof on the surface of the chip. The problem of this approach is that it requires a mixed design, which is much more complicated from a CAD perspective than a purely digital design. Also, the analogue parts are consuming a lot of power and area in the design. Those practical and economical reasons explain why the analogue solution is obsolescent.

Therefore modern designs resort to all-digital detection mechanisms. The generic ones exploit some artificial redundancy. It can be either implemented in time, space or information (code-based). All those strategies have been compared in [50], and shown to be roughly alike. Depending on the cryptographic scheme to protect, some dedicated countermeasures can also be implemented. The idea is to exploit some identities of the algorithm to protect so as to detect possible errors with a high probability. For example, in a typical encryption: the encrypted message can be decrypted and tested against the original plaintext. The same applies to digital signatures: the signature can be verified before being outputted. We wish to underline that these very verifications can represent a weakness per se, notably in front of so-called safe errors attacks [86].

However, the resilience against faults attacks has seldom been proposed. At the opposite, resilience in observation attacks is definitely a hot topic. Following the proposal of Paul C. Kocher made at the rump-session of CHES $2006^{1}$ [41] to update the keys on a frequent and regular basis, ideas for side-channel attacks resilient schemes have come up, as illustrated for instance by the "Provable Security against Physical Attacks" workshop [48]. But, to our best knowledge, no investigation about resilience against fault injection attacks has been published so far. Actually, many techniques of reliability have been ported as such to security applications. Nonetheless the objectives of reliability and security do differ:

- Reliability requires ideally that either the computations are correct or that an alarm is raised;

- Security requires that the computation result, if erroneous, carries no information about secret involved in the computation. This is a more flexible requirement than for reliability. On the one hand, it allows the system to output a false result $C^{*}$ instead of the correct one $C$, as long as it reveals

\footnotetext{
${ }^{1}$ Available on the CHES 2006 website.
} 
no information about the secret $K$. A formalization of security models under fault attacks can be done, for instance taking example on the practiceoriented framework [78] in the sibling case of SCAs. Actually this work has already been initiated for instance by this preliminary paper [46]. From an information-theoretic perspective, the requirement can be stated as "the mutual information between $\left(C, C^{*}\right)$ and $K$ is null'. On the other hand, rising an alarm can even be a vulnerability in some contexts. For instance, the differential behavior analysis (DBA [71]) manages to extract a key simply by knowing whether or not the computation went well, provided the fault model is of "stuck-at" type and roughly reproducible. FIR has no concept of alarm, hence is immune against such attack methods.

Therefore, in this paper, we challenge the reflex of transposing methods of reliability to security, because we prove that they are overly conservative.

\section{B. Comparison between Detection and Resilience}

Neither detection nor resilient schemes are able to withstand all the faults. Indeed, whatever the protection mechanism, we can theoretically build an attacker (possibly adaptative) able to replace an authentic value with another one. The goal of the countermeasure is to make this substitution very chancy.

In this subsection, we investigate the side-effects of the countermeasures. The detection strategy suffers two drawbacks illustrated in Tab. I. First of all, the device can raise an alarm even if the result is correct. This is the case when the fault happens on a variable that does not impact the output. This situation is of course not true in general, otherwise the variable could have been removed from the implementation. However, in the course of a specific computation, this is indeed possible. One trivial example is the result of an AND gate, that has zero for one input, and that is faulted on its second input. The fault will not be propagated and the result will be correct irrespective of the fault taking place or not. However, if a detection mechanism raises an alarm, then the whole computation will be stopped and adequate actions will be undertaken, thus causing a denial of service (DoS) despite the absence of any security problem. The DoS can also be seen as an attack path, where the opponent's goal is simply to prevent the cryptosystem from functioning. Also, the detection process in itself can be threat. Unless implemented in a discrete manner, the detection process can be spied by the analysis of a side-channel [39, Chp. 2], thus opening
Table I

CLASSICAL FAULT DETECTION CHARACTERISTICS, WHERE INCONVENIENT FEATURES HAVE BEEN HIGHLIGHTED IN RED COLOR.

\begin{tabular}{|c|c|c|c|}
\hline & \multicolumn{2}{|c|}{ Ciphertext incorrect? } \\
\hline & & Yes & No \\
\hline \multirow{2}{*}{ 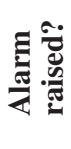 } & $\stackrel{\theta}{\nu}$ & Safe & $\begin{array}{l}\text { Problem of } \\
\text { availability }\end{array}$ \\
\hline & 之 & $\begin{array}{l}\text { Problem of } \\
\text { security }\end{array}$ & Safe \\
\hline
\end{tabular}

the door to attacks exploiting hypothesis testing (e.g. safe errors [86]). Such attacks require nevertheless a lot of care, since the attacker must be able to cut the power of the system before it erases its secrets. The second drawback of detection mechanisms is that they do not cover all the possible faults, and some faults can propagate without being detected.

On the contrary, an ideal resilient scheme will feature:

- an optimal availability: false detections do not exist, since errors are not caught but propagated.

- an optimal security: the fault generates a wave of erroneous data independent of the previous pristine (and sensitive) values. Therefore no sensitive information is propagated.

Also, in terms of coding and deployment guidelines, the advantages of resilience as opposed to resistance (fault detection) are manifold. We can really claim that resilience is a new security approach to protect cryptography, because of these typical improvements:

- In traditional designs, miscellaneous checks are scattered in the code. For instance, ratification counters and baits are usual tricks to detect "blind attacks". No such extra operations are required in the context of fault resilience, since it is not catastrophic that the IC fails. To be perfectly clear, such subterfuges are more palliative than curative. They notably hinder automatic or formal code expertise, although some applications would demand such a high confidence evaluation level.

- When using detection, faults can also occur in the detection logic. But then, the problem becomes eventually insolvable, since more and more logic is necessary (by recursion, we need detection logic for the detection logic, itself being protected by detection, etc.)

- On top of that, the resilience relieves the designer from having to deal with the reactions to the threat. These features are all in one very annoying for the 

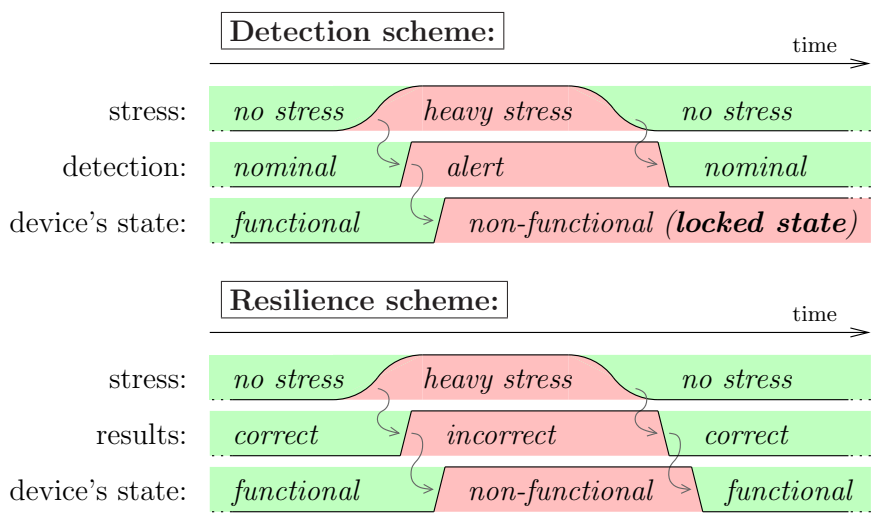

Figure 1. Suicide in case of fault detection (top), opposed to survival in case of fault resilience (bottom) protection schemes.

chip manufacturer; if they are activated unexpectedly they possibly ruin the device, causing large costs to replace the defective card. Now, the secure chip manufacturers are often balancing between activating the maximum level of countermeasures and risking card auto-scuttling (false positive) ${ }^{2}$. Such a dilemma does not exist with fault resilience. The card starts to produce faulty results while under stress (either because of an attack or because of a natural hazard), but returns to its nominal operating conditions as soon as the stress disappears. Thus the risk of have a permanent damage due to a false alarm is merely nonexistent. This point is exemplified in Fig. 1.

\section{Further Merits of the FIR}

One feature that gives to FIR a remarkable strength is its agnosticism with respect to attacks. By making any faults independent at its source and during its propagation independent of the previous values, it merely prevents any attack at its root. Therefore, new scenario schemes not envisioned yet are thwarted proactively, which provides a forward security. Typically, most if not all - attacks studied so far are differential: they assume the attacker knows couples of correct \& faulted computations corresponding to an identical (and presumably unknown) plaintext. Now, higher-order attacks

\footnotetext{
${ }^{2}$ Remember that early countermeasures against faults were intended to make up for the poor quality card readers, that inappropriately injected unwanted electrical glitches in the smartcards! Also, Ross Anderson and Markus Kuhn explained in [3] that the wild fluctuations in clock frequency that frequently occur when a card is powered up and the supply circuit is stabilising, caused so many false alarms that the [detection] feature is no longer used by the card's operating system.
}

could as well be possible: they would imply more than one faulty result. Additionally, faulted ciphertext-only attacks could also be devised. FIR fights all those future new threats that a pure DFA counter-measure would maybe fail to cover.

\section{Related Works}

Earlier publications have noticed the interest of allowing cryptographic devices to output faulty results, without jeopardizing their security. However, all those results focused on asymmetric cryptography, and more specifically on RSA. A fault tolerant RSA with CRT ${ }^{3}$ algorithm is given and formally proved in [87]. This article introduces the concepts of "fault infective CRT computation" and "fault infective CRT recombination". the algorithm is designed to have the errors occurring during the "mod $p$ " half propagate in the "mod $q$ " half, and vice-versa, thus denying the Bellcore [14] attack. This idea is definitely a FIR, albeit crafted to the case of RSA and more specifically against the Bellcore attack, whereas in our paper, the FIR is algorithm-agnostic.

Other formal ways to secure sensitive algorithms have been proposed. For instance, the paper [26] about "Algorithmic Tamper-Proof" (ATP) explains how to protect an implementation, by the specification of security requirements on the circuit and by restricting the power of the attacker. A cryptographic module implementing the FIR is definitely not protected in the context described in paper [26]. We would like to make clear that the FIR notion introduced in our paper applies to a system that has a trusted environment: the asset at risk is therefore only the cryptographic core. In other terms, the two methods ATP and FIR do not consider the same security boundary.

\section{Some Practical ImPlementations of FIR}

The purpose of this section is to provide with some actual instances of resilient cryptographic schemes. For the sake of clarity, we focus on the protection of symmetric block encryption modules. Indeed, as they are deprived by construction from any algebraic properties, they are also the most difficult ones to protect. The stateof-the-art in asymmetrical algorithms protection is very well advanced and formally proved. An overview, on the example of RSA, can for instance be found in these papers [15], [16].

\footnotetext{
${ }^{3}$ The computations "mod $n=p \cdot q$ " are done separately "mod $p$ " and "mod $q$ ", and then combined back. This processing - possible only for the owner of the private key - speeds up the overall computation by a factor of four.
} 
In the subsection III-A, we present a FIR approach that works at high-level, on top of an unprotected cryptographic module: it is a protocol-level resilient scheme. The subsection III-B rather introduces two solutions at the gate-level, where FIR is intricated with the cryptographic module's implementation. In those two embodiments of FIR, we assume the cryptographic parameters are loaded securely, and thus that key alteration attacks (see for instance [26] or $\S$ III.C of [6]) are out of the scope. To sum up, our security goal is definitely the protection of symmetric cryptographic operations.

\section{A. Formal Counter-Measures against Fault Injection At- tacks}

A differential fault analysis (DFA [13]) requires the same plaintext to be encrypted twice with the same key. Common attack scenarios consider the case where the attacker is able to inject one fault in only one of the encryptions. Then, she can deduce information about the key using a DFA. Thus, DFAs are made impossible if an attacker is not able to request twice the same encryption. It is possible to devise such a scheme, as typified by algorithm (1).

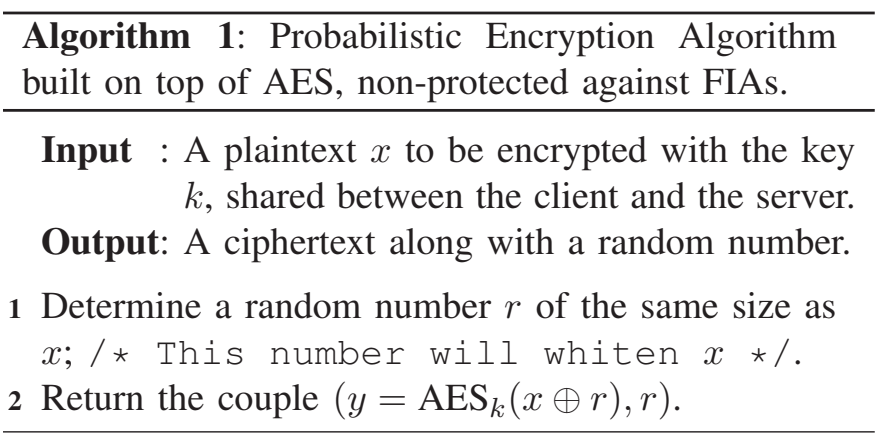

This algorithm (1) is considered as secure against DFA because the probability that two encryptions are generated with the same plaintext is roughly speaking $2^{n / 2}$, where $n$ is the entropy of $x$ or $r$. Indeed, this is a classical instance of the birthday paradox.

We mention additionally that the scheme of algorithm (1) protects against a broader class of attacks than only the DFAs. It is a random encryption scheme, that has the remarkable property that the attacker cannot decide if the encryption is actually faulty or not. Indeed, in an ideal block cipher, an attacker cannot distinguish between the outputs of that cipher and of a noise generator. Therefore, in the case of a random FIA, the attacker gets no additional information, hence no advantage, from her perturbations of algorithm (1). Thus, safe-error [86] attacks on the block cipher are also impossible: even if the attacker manages to inject a precise fault (in time, space and value) in the early rounds of the algorithm, there is no way for her to know from the encryption result whether this value is correct or not.

As a security notice, it must be understood that the protocol (1), used as such, can be forged. Indeed, if one authentic transaction is spied by an attacker, she gains access to a couple $\left(y=\operatorname{AES}_{k}(x \oplus r), r\right)$. Now, let us consider the case where the attack wishes to impersonate the client. It is straightforward, in front of a new request $m^{\prime}$ to return a valid encryption without knowing the secret key $k$. The imposter can simply choose maliciously the random variable $r^{\prime}$ as $r^{\prime}=m \oplus m^{\prime} \oplus r$, and return $\left(y, r^{\prime}\right)$, which is a valid encryption. Therefore, the protocol should include a challenge. For instance, the random variable $r$ can be sent from the server instead of being chosen by the client itself.

Unfortunately, this scheme is not secure in decryption. As a matter of fact, the decryption algorithm corresponding to (1) is given in algorithm (2). This algorithm can be called repeatedly without the AES inputs being modified: it is deterministic.

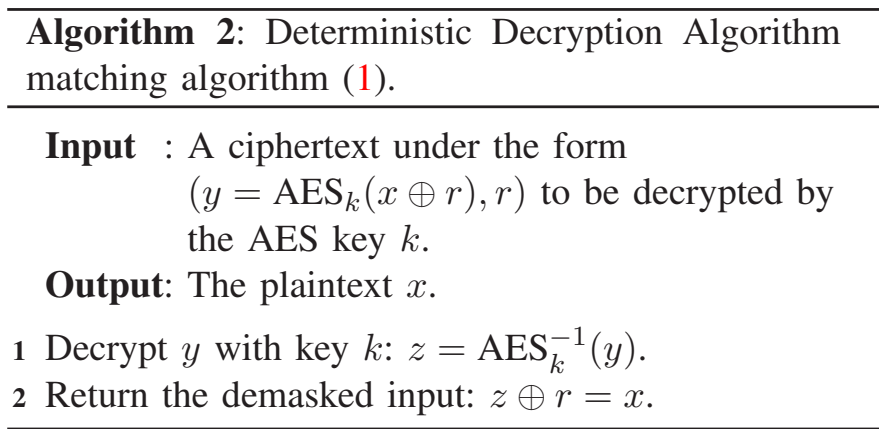

This situation can however be exploited to protect low cost embedded systems, such as smartcards or RFID tags, that communicate with a larger device, such as a reader. In this situation, there is a natural asymmetry between the two protagonists. This fact has been emphasized in other publications on lightweight embedded systems security, such as [29, §1]. It is fairly easy to protect the reader against fault attacks by "physical tamper-proof measures". For instance, the reader electronic circuits can be imprisoned into a mold, protected with a pasted metallic cover and sealed into a box equipped with intrusion detection sensors. The same level of sophistication is impossible for smartcard or tags modules, because their form factor is extremely constrained in size (due to stringent requirements about 


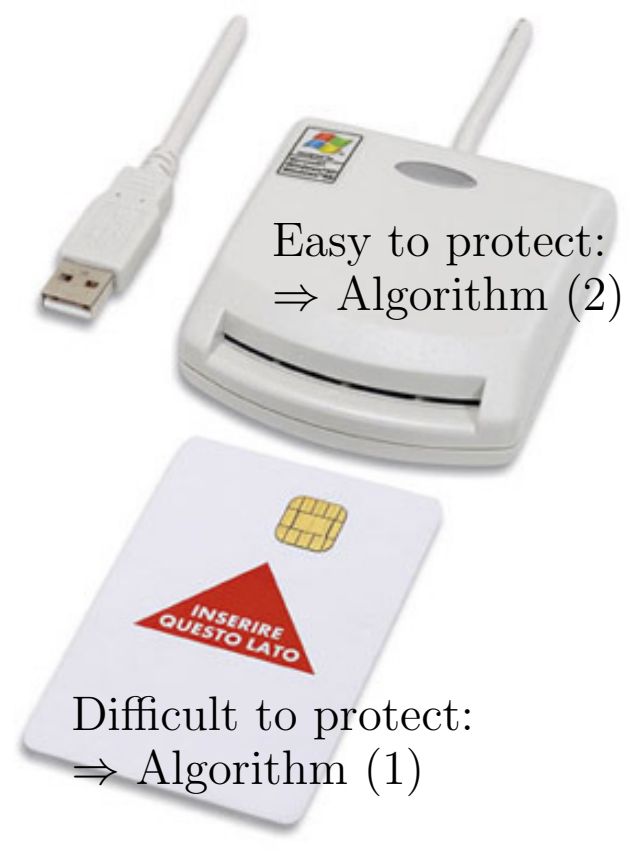

Figure 2. Probabilistic encryption is performed on the most vulnerable device while the deterministic decryption is safely carried out within the most secure device.

the mechanical strength edicted by standard ISO 78161). Hence ways to attack smartcards are - unfortunately very numerous [43]. Additionally, smartcards are cheaper to buy than readers, and, to top it all, the selling of smartcards is necessarily less restricted than that of readers, because in any deployment context, there are more smartcards out than card readers. Therefore, the attacker will most certainly prefer to attack the embedded system to extract the shared secret key. Thus, if the reader plays the decryption (2) and the embedded system the encryption (1), the unbalance between the tamperresistance of the two devices is made up by the opposite unbalance of the algorithm, in terms of resistance against DFA. This strategy of reinforcing the security by algorithmic means of the weakest element in the security chain is illustrated in Fig. 2.

Notice that if a handy homomorphous encryption algorithm HEA is available, a completely secure encryption/decryption scheme can be devised. Let us denote by $\mathrm{HDA}=\mathrm{HEA}^{-1}$ the corresponding decryption algorithm and $\times$ the composition law in the group of homomorphy:

$$
\forall y_{1}, y_{2}, \quad \operatorname{HDA}\left(y_{1} \times y_{2}\right)=\operatorname{HDA}\left(y_{1}\right) \times \operatorname{HDA}\left(y_{2}\right) .
$$

The encryption proceeds as per algorithm (1) using HEA instead of AES, whereas the decryption consists in algorithm (3). This scheme can use for instance Paillier's cryptosystem [63] as underlying encryption primitive. However care must be taken with RSA [14].

Algorithm 3: Probabilistic Decryption Algorithm matching (1) with HEA instead of AES as underlying cipher.

$$
\begin{gathered}
\text { Input : A ciphertext under the form } \\
\left(y=\operatorname{HEA}_{k}(x \oplus r), r\right) \text { to be decrypted by } \\
\text { the HEA key } k .
\end{gathered}
$$

Output: The plaintext $x$.

1 Determine a random number $s$ of the same size as $y$ or $r$.

2 Return $\operatorname{HDA}_{k}(y \times s) / \operatorname{HDA}_{k}(s) \oplus r=x$.

The resilient algorithms presented in this subsection III-A have the drawback that the size of the ciphertext is doubled. This can be a limitation for instance in contactless cards authentication, where the transmission time must remain short. Also in wireless sensor network the increase of the data transmitted means a very high cost in term of power.

Nonetheless the algorithm (1) can be made more bandwidth and power-efficient if the message $x$ to encrypt is cut in several blocks. In this case, alternative encodings, such as the probabilistic all-or-nothing transform (AONT) described in [54], [53], could be taken advantage of. This paper and this patent introduce a probabilistic symmetric encryption algorithm, in a view to thwart SCAs. With respect to other probabilistic symmetric encryption scheme (most of the times, the encryption involves a random IV - which is short for initialization vector), this AONT scheme is original in the sense that the randomness is not disclosed along with the ciphertext. This denies the possibility to conduct a side-channel attack on the first round(s) of the encryption algorithm. A similar scheme has also been described in [55]. As such, this all-or-nothing scheme (in general, but also under the form of its "Probabilistic Signature Scheme", aka PSS, avatar [19]) is an implementation of FIR. In addition, it reduces the number of blocks to be exchanged to the number of plaintext blocks plus one. In summary, algorithm (1) combined with [54] has the benefit of bringing a SCA-resistance in addition to the FIA-resilience. Certainly, this suggestion of protocollevel countermeasure can be optimized, but we leave this topic open for future works [10]. 


\begin{tabular}{|c|c|c|c|c|c|c|c|c|c|}
\hline \multicolumn{4}{|c|}{ Multi-valued logic } & \multicolumn{6}{|c|}{ Redundant logic } \\
\hline \multicolumn{2}{|c|}{ Case \#1 } & \multicolumn{2}{|c|}{ Case \#2 } & \multicolumn{3}{|c|}{ Case \#1 } & \multicolumn{3}{|c|}{ Case \#2 } \\
\hline & & & & & & & & & \\
\hline 1 & 1 & 1 & & & & & & & $\square^{*}$ \\
\hline $1 / 2$ & $\zeta^{1 / 2}$ & $1 / 2$ & $>1 / 2$ & 01 & & 0 & 01 & & 10 \\
\hline & $\begin{array}{ll}P^{*} & 0 \\
\end{array}$ & & $\mathbb{7}$ & $*$ & & & & & * \\
\hline
\end{tabular}

Figure 3. Two kinds of faults (in red), namely $\{0,1\} \stackrel{*}{\rightarrow} 1 / 2$ for 3 -valued logic and $\{01,10\} \stackrel{*}{\rightarrow}\{00,11\}$ for DPL, after which the initial value (in green) has been forgotten.

\section{B. Multi-Valued and Redundant Representation Logics}

Multi-valued logics allow to encode more than one bit with one electrical state. It is for instance used in some power-constant logic styles [5]. Let us consider the case of an equipotential holding three states, denoted 0 , $1 / 2$ and 1 , amongst which only the two 0 and 1 are functional. Then, if a fault turns a valid value into $1 / 2$, the provenance state (either 0 or 1 ) has been forgotten.

The same goes for redundant logics, such as the $m$ out-of- $n$ representations (for $0<m<n$ ). For instance, the 1-out-of-2 representation, also known as dual-rail with precharge logic (DPL), admits two valid states, denoted by 01 and 10, and two invalid states, denoted by 00 and 11 . In the case one fault turns a valid token into an invalid one, the value before the fault is lost. The effect of faults on these two logic styles is summed up in Fig. 3. It clearly appears that the state after the fault is decorrelated from the initial state, thereby establishing the resilience, for the relevant cases where the data is sensitive.

This protection mechanism is nonetheless less powerful than that based on input message randomization. Indeed, by merely looking for invalid tokens in the output, the attacker can decide if a fault has been having an effect. Without loss of generality, let us take the example of DPL where the spacer token used for precharge is 00 . One typical fault scenario is the valid tokens not having enough time to evaluate. Hence, some tokens supposed in theory to get regular value 01 or 10 remain in practice stuck at idle value 00 . If the ciphertext has an abnormally low Hamming weight, the attacker can deduce that, with a high probability, a fault has been injected successfully and has propagated. Thus redundant logic styles are not secure against all kinds of attacks that do not exploit the faulted result, but merely the behavior (faulty or not). This concerns the safe-errors attacks [86], the DBA [71] and the FSA [47]. Only DFAs are thwarted, because the value of the faulted ciphertext is unrelated to the netlist internal secrets.

Now, the resilience only works in the case the attacker fails to inject "valid false" faults, i.e $0 \stackrel{*}{\leftrightarrow} 1$ faults in multivalued logic or $01 \stackrel{*}{\leftrightarrow} 10$ faults in DPL. Let us assume, for the moment ${ }^{4}$, that this situation is rare. It seems all the more difficult to achieve in DPL because the attacker must produce two antinomic concerted faults.

As will be exposed into greatest details in Sec. IV, the resilience will build up each time a valid false is produced along with invalid faults. In this case, the two faults will propagate, and if the logic favors the generation of invalid instead of valid states, then the diffusion of the netlist will encourage the invalid states to hide the false valid states. This case is optimal if the logic meets this requirement:

"if any input is invalid, so is the output".

This behavior is "saturating"; the faults will percolate in the netlist and the invalid values will saturate most of the nets, thereby absorbing all the false valids that are crossed. So the resilience is amplified by the diffusion in the netlist and the collaborative behavior of gates to favor invalid values propagation. This phenomenon of invalid values (dominant) suppressing false valid values (recessive) is further detailed in the next section IV.

\section{Dual-Rail with Precharge Logic as a Global Countermeasure against IMPLEMENTATION-LEVEL ATTACKS}

DPL styles are solutions primarily designed to protect a cryptographic implementation against side-channel attacks. However, it has been noticed that these styles can also natively withstand some perturbation attacks [57], [58], [73], [11]. It has already been underlined in Sec. II that, unlike traditional counter-measures against fault attacks, the DPL does not implement a protection, but is rather resilient. This means that faults are not caught, but rather left free to cascade their effect, knowing that eventually their observable consequences will not be harmful from a security standpoint.

\section{A. Requirements for Simultaneous SCA and FIA Protec- tion}

In order to better illustrate the close relationship between observation and perturbation attacks, we need to notice that security perimeters depend on the application. For instance, in an ISO/IEC 7816 compliant smartcard, several security violation situations can be encountered.

\footnotetext{
${ }^{4}$ A study of "valid false" survival conditions is provided in Sec. IV-E.
} 


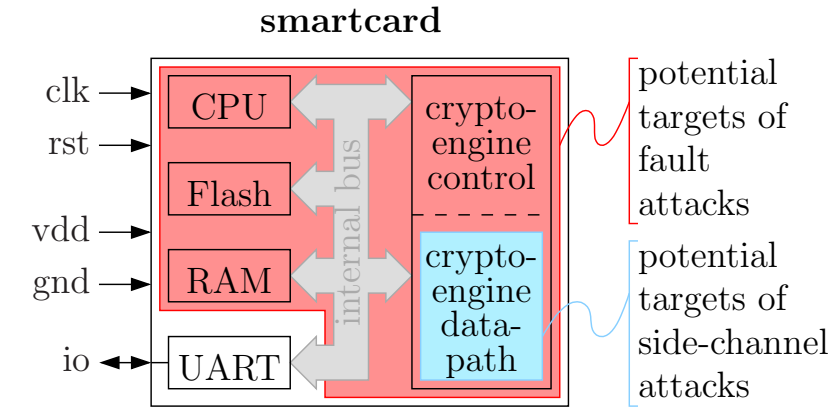

Figure 4. Susceptible organs of a smartcard in two representative sensitive operations (EXTERNAL AUTHENTICATE and INTERNAL AUTHENTICATE). Typically, the cryptography will be triple-DES or AES, i.e. the most widely industrially adopted block ciphers.

- The critical part is the memory in case of an external authentication. Indeed, if the memory can be corrupted, then any rogue reader can be forced to be seen as authentic. Here, there is no secret to retrieve, but simply an invalid state to be setup by force.

- However, during an internal authentication, the smartcard uses its cryptographic secret. Therefore, the risk for the smartcard is to have its key retrieved illegitimately. Differential fault attacks and sidechannel attacks are two tools available to recover the key. In addition, as the protection against attacks is costly, the designer will try to partition the cryptographic block at risk. Typically, when he implements symmetrical encryption, this block can be split into:

- a control part, subject to fault attacks, such as round reduction attacks [56], but leaking no sensitive information as the algorithm is supposed to be known by the attacker (common assumption with Kerckhoffs' law), and

- a data processing part, subject to both fault attacks, such as DFAs [13], [64], and sidechannel attacks, such as DPA [42].

The overall requirement for security against implementation-level attacks in a smartcard is depicted in Fig. 4. This block-diagram shows in red the security boundary for fault attacks and in cyan that for SCAs. It appears clearly that some organs shall be protected only against fault attacks, but that all the organs that shall be protected against SCA must also be protected against FIA. This is an advanced question, all the more important as it is in this part of the design that the largest overheads are expected.
The countermeasures against SCA include:

- information hiding, implemented with DPL,

- information masking, implemented with random splitting of data into shares.

More information about these two categories of protection against SCAs can be found in the "DPA book" [51], respectively at chapter 7 and 9. Amongst this array of possible protections, DPLs [77], [21] are of particular interest because they have native protections against DFAs. We will thus focus in the rest of this article on the combined DFA and SCA protection of the datapath of cryptographic modules; The type of fault attacks we consider are those described in [27], the two most famous of them being that of Biham \& Shamir [13] (DES [61]) or Piret \& Quisquater [64] (AES [62]), enhanced by Tunstall in [84]. Another motivation to focus on the crypto-datapath is that it is usually the most complex design part; therefore it represents the largest area of the design and contains the longest critical timing paths. This explains that local faults are more likely to target the datapath because of its predominant surface, and that global faults also affect preferentially the datapath that is most tight when it comes to meeting the setup time constraint.

\section{B. Previous Art about DPL in the Presence of Faults}

We use the following notations for the DPL representation. Every logical variable $a$ is represented by a couple $\left(a_{f}, a_{t}\right)$ of wires, that carry two values. The term $a_{f}$ (resp. $a_{t}$ ) is short for the proposition " $a$ is false, i.e. $a=0 "$ (resp. " $a$ is true, i.e. $a=1$ "). The semantic of the four possible combinations is detailed below.

- $a$ is VALID if $a_{f} \oplus a_{t}=1$. More precisely, VALID $\doteq\{$ VALID0, VALID1 $\}$ or, more explicitly, VALID $\doteq\{(1,0),(0,1)\}$

- $a$ is NULL if $a_{f} \oplus a_{t}=0$. More precisely, NULL $\doteq\{$ NULL0, NULL1 $\}$ or, more explicitly, $\mathrm{NULL} \doteq\{(0,0),(1,1)\}$.

The two NULL states are used alternatively with the VALID ones as precharge stage, so that the next evaluation starts afresh from a known state. The DPL protocol is recalled in Fig. 5.

There are two flavors of DPL, depending on whether they feature the early propagation effect (named EPE in the literature, and incidentally discovered independently by [79] and [44] in the same year) or are protected against it. The definition of those variants can be summarized by the following conditions to be fulfilled by all the instances $f$ : 


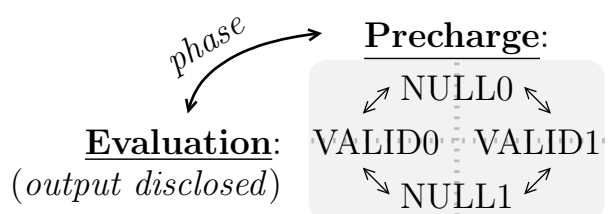

Figure 5. DPL protocol. Meaning values are computed during the evaluation stage; the precharge is meant to setup the nets to a known and data-independent initial state.

- DPL w/ EPE: $\exists a$ VALID, $f(a$, NULL $)=$ VALID; - DPL w/o EPE: $\forall a$ VALID, $f(a$, NULL $)=$ NULL.

To be properly protected against SCAs, those logics must be balanced at the layout-level [83], [34], [31], to preserve indiscernibility properties (typically true $\leftrightarrow$ false symmetry). Otherwise, straightforward attacks that exploit the physical unbalance become possible [72]. However, when analyzing those logics regarding FIR, the physical layout can be forgotten, and only the logical functions are considered.

In DPL, only results on evaluation are observable, because return to precharge faults are not outputted. We adopt the following faults typology on DPL:

- Asymmetric faults: \{VALID0, VALID1\} $\stackrel{\downarrow}{\longrightarrow}$ NULL0, triggered by global perturbations (e.g. caused by a setup time violation due to power/clock glitch, overclocking or under-powering);

- Symmetric faults: \{VALID0, VALID1\} $\stackrel{\downarrow \text { or } \uparrow}{\longrightarrow}$ \{NULL0, NULL1\}, triggered by local perturbations (e.g. caused by injection of high energy laser light, electromagnetic field or particles beam).

1) DPL w/ EPE is Protected against Multiple Asymmetrical Faults: WDDL [82] is a typical DPL w/ EPE style. In this logic, the AND function is defined as: $\left(y_{f}, y_{t}\right) \doteq\left(a_{f}+b_{f}, a_{t} \cdot b_{t}\right)$. We use the following color code in Boolean truth tables:

- gray: the regular truth table in the absence of faults (i.e. the intended functionality),

- purple: anticipated values (evaluation even if not all inputs are valid).

Otherwise, the green and red colors still represent respectively correct and incorrect behaviors or properties.

As shown below, WDDL can propagate correct valid results in the presence of asymmetrical faults.

\begin{tabular}{|c|ccc|}
\hline$b$ & VALID0 & VALID1 & NULL0 \\
\hline VALID0 & VALID0 & VALID0 & VALID0 (EPE) \\
VALID1 & VALID0 & VALID1 & NULL0 \\
NULL0 & VALID0 (EPE) & NULL0 & NULL0 \\
\hline
\end{tabular}

This behavior is positively resilient. It is that of the Unitialized value in VHDL enumerated type ieee.std_logic_1164.std_ulogic, recalled below:

\begin{tabular}{|c|c|c|c|}
\hline$b \quad$ & ${ }^{\prime} 0^{\prime}$ & $' 1^{\prime}$ & ${ }^{\prime} \mathrm{U}^{\prime}$ \\
\hline$' 0^{\prime}$ & ${ }^{\prime} 0^{\prime}$ & ${ }^{\prime} 0^{\prime}$ & ${ }^{\prime} 0^{\prime}$ \\
\hline$' 1^{\prime}$ & ' $0^{\prime}$ & ${ }^{\prime} 1^{\prime}$ & ${ }^{\prime} U^{\prime}$ \\
\hline${ }^{\prime} \mathrm{U}^{\prime}$ & $0^{\prime}$ & ${ }^{\prime} U^{\prime}$ & ${ }^{\prime} U^{\prime}$ \\
\hline
\end{tabular}

where the tokens \{VALID0, VALID1, NULL0\} implement respectively the items $\left\{{ }^{\prime} 0^{\prime},{ }^{\prime} 1^{\prime},{ }^{\prime} U^{\prime}\right\}$.

These conclusions can be challenged in the case of a coupling of the fault injection analysis with a sidechannel analysis. For instance, the fault sensibility analysis (FSA [47]) can, under some circumstances, exploit the unbalance within the two wires making up a dualrail pair. However, the FSA has only been demonstrated as partially successful on a WDDL chip; and WDDL is known to be extremely unbalanced [72].

Actually, this FIA-resistance solution has already been sketched in [38]. This article introduces two methods to protect circuits against FIAs.

The first one consists in resisting to an arbitrary number of "stuck-at-0"5. Those "reset faults" correspond to our "asymmetric faults". However, this publication is overly conservative; invalid tokens are generated even if the data is not tainted. Also, the authors of [38] add a series of cascade gates at the output of the circuit. Their role is to turn all other valid tokens to invalid ones. Additionally, they request that the circuit commits suicide at this point (when the ciphertext is all NULL, noted " $\perp$ " in [38]). Our key remark is that those two requirements are actually overkill. Indeed, the overall security is not jeopardized if some valid and some invalid tokens are outputted; therefore, we can save the cascade stage. In addition, we insist that it is then useless to permanently destroy the circuit: as we know the attacker only gets faulted crypto results that do not convey any information about the sensitive variables, it is safe to continue without erasing the secrets, that are merely not compromized. Therefore, the scheme we present is more user-friendly, in the sense it keeps the application up-and-running unless a fault is indeed influencing the result.

The second countermeasure against arbitrary faults in [38] is more ad hoc, since one needs to know the maximum number of faults an attacker can inject to dimension the level of protection (based on an adaptively

5 ...or equivalently "stuck-at-1" for all the faults. 
sized countermeasure). In the next paragraph, we study FIR in the presence of multiple symmetric faults.

2) DPL w/ EPE is not Protected against Multiple Symmetric Faults: To start with, we assume neither $a \stackrel{*}{\rightarrow} \bar{a}$ nor $b \stackrel{*}{\rightarrow} \bar{b}$ happens. However, even in this favorable case, WDDL can generate incorrect false results. They are presented by skulls (symbol: in the following table.

\begin{tabular}{|c|cccc|}
\hline$b$ & VALID0 & VALID1 & NULL0 & NULL1 \\
\hline VALID0 & VALID0 & VALID0 & VALID0 (EPE) & VALID0 (EPE) \\
VALID1 & VALID0 & VALID1 & NULL0 & NULL1 \\
\hline NULL0 & VALID0 (EPE) & NULL0 & NULL0 & VALID0 ( 8 ) \\
NULL1 & VALID0 (EPE) & NULL1 & VALID0 (8) & NULL1 \\
\hline
\end{tabular}

For instance, the twain simultaneous errors:

1) $a=$ VALID $1 \stackrel{* \uparrow}{\longrightarrow} a=$ NULL1 and

2) $b=$ VALID $1 \stackrel{* \downarrow}{\longrightarrow} b=$ NULL0

trigger a dreadful transformation: VALID1 $\stackrel{*}{\longrightarrow}$ VALID0.

Therefore, because of EPE, logical inversions $f(a, b) \stackrel{*}{\rightarrow} \overline{f(a, b)}$ can occur, which makes FIAs (such as DFAs) possible.

3) DPL w/o EPE is Protected in front of Multiple Symmetric Faults: Now, the DPL w/o EPE styles are protected against multiple symmetric (hence asymmetric) faults. This is shown in the table below.

\begin{tabular}{|c|cccc|}
\hline$b$ & VALID0 & VALID1 & NULL0 & NULL1 \\
\hline VALID0 & VALID0 & VALID0 & NULL0 & NULL1 \\
VALID1 & VALID0 & VALID1 & NULL0 & NULL1 \\
NULL0 & NULL0 & NULL0 & NULL0 & NULL1 \\
NULL1 & NULL1 & NULL1 & NULL0 & NULL1 \\
\hline
\end{tabular}

Remark that if we call:

- '0': VALID0,

- ' 1 ': VALID1,

- ' $\mathrm{X}^{\prime}:$ NULL $=\{$ NULL0, NULL1 $\}$,

then we have the same behavior (i.e. "propagate always") as VHDL. This is illustrated below:

\begin{tabular}{|c|c|c|c|}
\hline$b$ & $' 0^{\prime}$ & ${ }^{\prime} 1^{\prime}$ & ${ }^{\prime} X^{\prime}$ \\
\hline${ }^{\prime} 0^{\prime}$ & ${ }^{\prime} 0^{\prime}$ & ${ }^{\prime} 0^{\prime}$ & ${ }^{\prime} X^{\prime}$ \\
\hline${ }^{\prime} 1^{\prime}$ & ${ }^{\prime} 0^{\prime}$ & ${ }^{\prime} 1^{\prime}$ & ${ }^{\prime} X^{\prime}$ \\
\hline${ }^{\prime} \mathrm{X}^{\prime}$ & ${ }^{\prime} X^{\prime}$ & ${ }^{\prime} X^{\prime}$ & ${ }^{\prime} \mathrm{X}^{\prime}$ \\
\hline
\end{tabular}

Finally, we note that even if a few mutations $a \stackrel{*}{\rightarrow} \bar{a}$ exist for some variables $a$, it is very likely that the ' $\mathrm{X}$ ' wave caused by $a \stackrel{*}{\rightarrow}$ NULL eats them. As detailed in the next sub-section, the recessivity of ' $X$ ' over NULL, coupled with the avalanche of ' $X$ ' caused by the diffusion property of the logic, accounts for that.

\section{Detection scheme:}

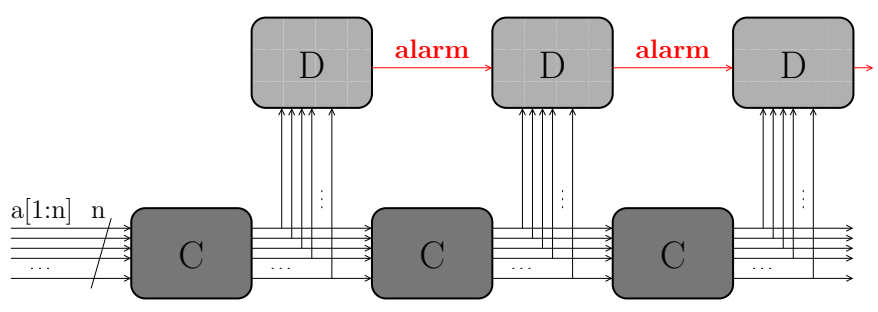

\section{Resilience scheme:}

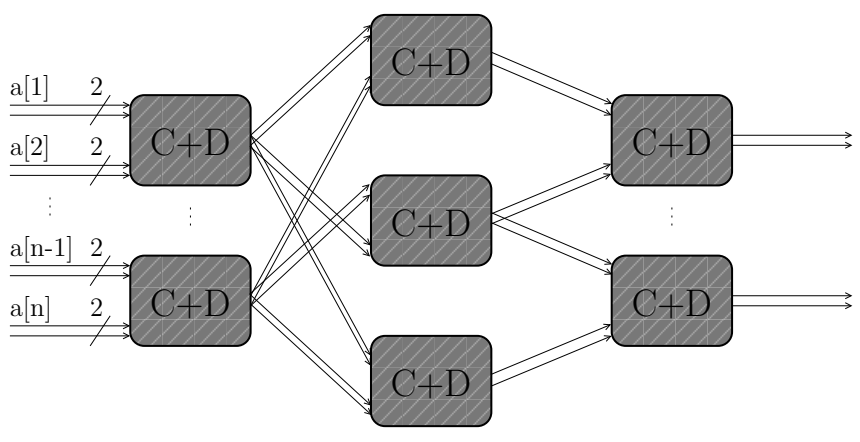

Figure 6. Difference of detection and resilience working factors, represented on an example netlist.

\section{Revisiting the Comparison Resilience vs. Detection}

One can argue that the DPL used as a FIR is in fact a very low-grain fault detection scheme. Indeed, FIR shares with the detection strategy the fact that redundancy is required. However, it is coupled to a diffusion that makes the detection at one stage take advantage of the rest of the stages. This detection is propagated in a wave, that constitutes a collaborative strategy that is absent from the pure detection schemes. This difference is illustrated in Fig. 6. In traditional detection schemes, the computation (noted: C) and the detection (noted: D) logics are dissociated. In particular, the detection blocks do not communicate. In the DPL FIR scheme, the computation and the detection are merged (noted: $\mathrm{C}+\mathrm{D}$ ) and this information propagates downwards the netlist.

There are two properties of DPL that help resilience:

- The redundancy of the netlist. At an $n$-bit output of a combinational block, only $2^{n}$ amongst the $2^{2 n}$ possible ones are valid.

- The diffusion within the netlist, which is characteristic to the cryptographic algorithms. This property is especially true at the netlist level for logics free from EPE [11]. Indeed, the fanout of each gate is double w.r.t. separable logics such as WDDL [82]. 
Current detections schemes work independently of the computation and in a non-collaborative way. At the opposite, FIR consists in intricating the detection agents with the computation and to tightly interconnect them. The objective is to trigger a proliferation of tamperevidence logic markers (NULL tokens).

\section{Cost Estimation of FIR versus Traditional Ap- proaches}

The traditional approach to counteract implementation-level attacks is a composition. The recommendations formulate like this:

- first use detection schemes, that can be inserted early at the RTL of the algorithm [45];

- then map this FIA-aware RTL description into a SCA-proof logic style. Indeed, the detection logic manipulates sensitive variables, and might itself leak secrets [69]. Therefore, it deserves a protection against SCAs. In a similar fashion, the study reported in paper [49] confirms that gatelevel countermeasures against FIAs do not reduce the information leakage.

This implies that the overhead of the FIA and SCA countermeasures get multiplied.

A typical overhead for FIA countermeasures can be found in [50]. Let us consider the case of a non-linear code, such as [40], that is suited to detect multiple faults. Its overhead is $77 \%$ in area and $15 \%$ in throughput.

As such, those performance losses are more affordable than those required to thwart SCAs. For instance, WDDL incures an increase of 3.1 in area and 3.9 in throughput [81].

The combination of [40] and [81] results in an increase of 5.5 in area and 4.5 in throughput.

Those results are to be contrasted with the FIR approach using an EPE-proof DPL style. This style already merges FIA and SCA countermeasures. The reported overheads for two of those logics are given in Tab. II. It clearly appears that using a symbiotic SCA+FIA countermeasure is more efficient than combining two countermeasures one on top of each other.

We notice that those alternative "DPL without EPE" logics yield similar performances: DRSL [18] ${ }^{6}$, iMDPL [65], IWDDL [52], STTL [75], [76], SecLib [35], [32], [33], [36], WDDL w/o EPE [11], [12], BCDL [60], [20] and LBDL [89].

We also attract the reader's attention on the fact that asynchronous logics, especially the quasi-delay insensitive (QDI) style [57], [56], can be implemented

\footnotetext{
${ }^{6} \mathrm{DRSL}$ is however shown to have a built-in security flaw in [60].
}

Table II

PERFORMANCE OVERHEAD OF DIFFERENT SCA+FIA COUNTERMEASURES.

\begin{tabular}{|r||c|c|c|}
\hline \multicolumn{1}{|c||}{ Strategy } & Detection + DPL & \multicolumn{2}{c|}{ Resilience = DPL } \\
\hline Countermeasure & {$[40]+[81]$} & DRSL [18] & IWDDL [52] \\
\hline \hline Area & $5.49 \times$ & $2.56 \times$ & $4.34 \times$ \\
Throughput & $4.49 \times$ & $2.00 \times$ & $1.53 \times$ \\
\hline
\end{tabular}

in DPL [30]. Now, asynchronous logic is designed to remain functional irrespective of the environmental variations. Concrete work [88] on this topic had been carried out in the framework of the G3Card project [24]. However, the G3Card consortium only detects NULL1 as an error marker in a DPL protocol where the only allowed spacer is NULL0. This signalization is restrictive and do not consider propagation of errors; instead, an instantaneous detection is suggested, which seams hard to put in practice in real-time given that such checks shall be done for each and every gate of the design. Moreover, asynchronous QDI logics have a drawback in terms of resilience: each gate being sequential in nature (due to the necessary handshakes with the upstream fanin and downstream fanout gates), a fault can cause a deadlock, should the fault cause a protocol violation (i.e. the transitions depicted in Fig. 5 are not respected). To relieve the circuit from this deadlock, the asynchronous circuit shall be reset. Thus the resilience provided by an asynchronous circuit is in-between the two cases illustrated in Fig. 1. The card is not destroyed permanently, since a reinitialization relaunches it; however, the system must detect that the logic hung (perhaps with the help of a watchdog) in order to restart it. Despite of these discrepancies with the FIR concepts, we note that QDI still increases the number of situations where the circuit remains functional, while remaining "resilient" if the external conditions are too harsh.

Eventually, we wish to underline that these overheads are not that dramatic when contrasted with those encountered in other domains that also require dependability features. Typically, the avionic industry makes use of techniques such as triple modular redundancy (TMR) to thwart single event upsets (SEUs). An example of a memorization element in TMR style is given in Fig. 7. The amount of logic involved in this structure is by far larger than that required in the DPL counter-part, depicted in Fig. 8. This structure has two stages to accompany the evaluation $\leftrightarrow$ dynamic of the DPL protocol. We notably insist that such a construction is naturally 


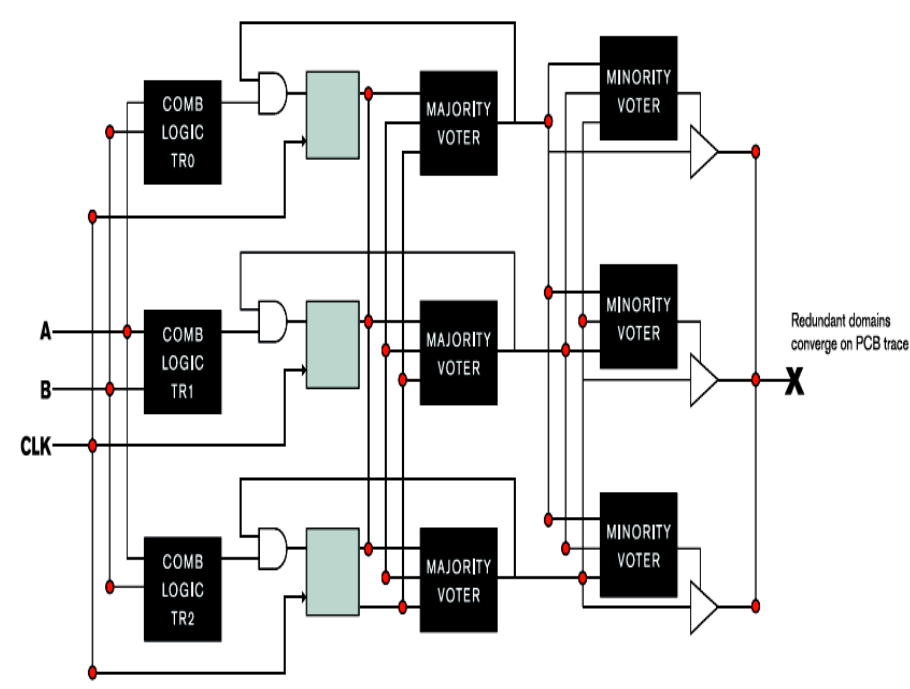

Figure 7. Memorization element in triple modular redundancy as implemented in Xilinx "XTMR" solution [80].

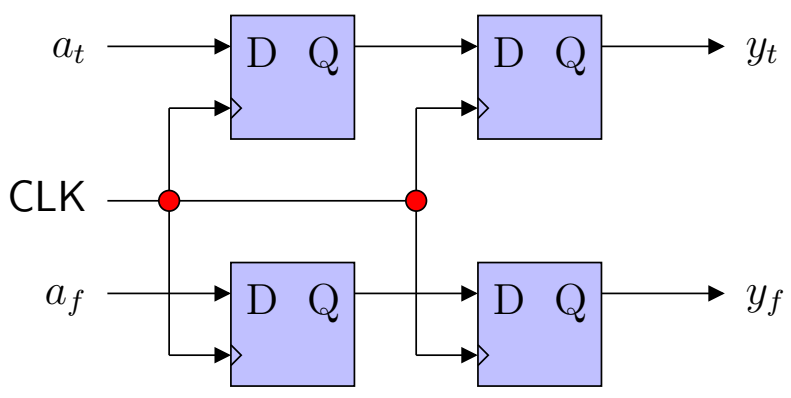

Figure 8. Memorization element in DPL; although four times larger than an unprotected flip-flop (DFF, represented as a violet square), this structure is nevertheless much smaller than that involved in TMR logic (see Fig. 7).

immune to the attack presented in [59], that exploits an optimization of some DPL style: when the redundant dual-rail state is stored as one single bit, an exploitable leakage appears at the flip-flop level. To conclude this comparison between figures 7 and 8 , we emphasize that the overhead figures shall not be considered in absolute, but relatively to the protection goal that is intended to be achieved.

\section{E. Associating Three Protections to Reduce the Proba- bility of a Successful FIA}

Some faults in DPL circuits do not disclose any information about the faulted sensitive variable. However, in the case false valid are generated, the problem becomes different. This can happen in two problematic cases:

1) When the absorbing fault is too deep in the logic cone w.r.t. the false valid, as shown in Fig. 9,

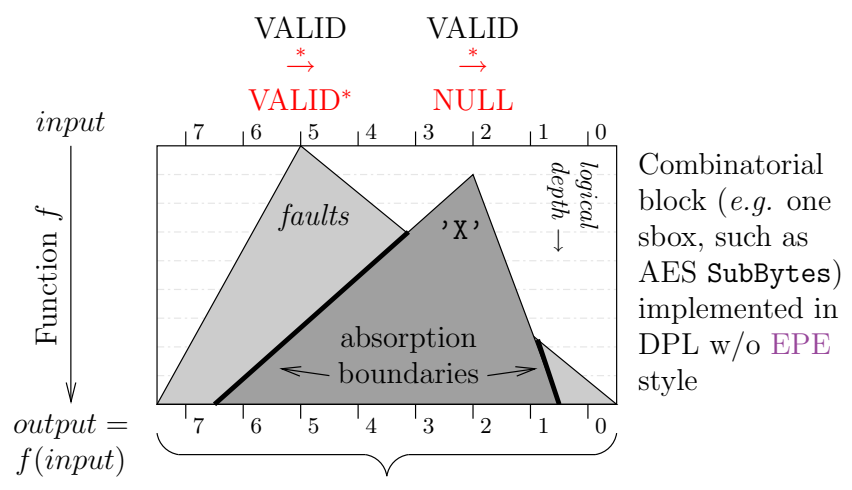

The output is mixed NULL and VALID*

Figure 9. Multiple faults, where the false valid is not completely hidden by the ' $\mathrm{X}$ ' wave. The ' $\mathrm{X}$ ' avalanche absorbs most, if not all, the valid faults.

where $f$ is a block with perfect ${ }^{7}$ diffusion, such as a substitution box implemented in logic. In this case, if the logic cone covered by the ' $X$ ' happens to yield a correct value, then a valid fault is generated; unless the ' $X$ ' are checked for at the output.

2) When a valid false occurs on one column alone, but that an ' $\mathrm{X}$ ' is generated on another column (knowing the two columns are not interfering in AES last round). In this case also, the faulty behavior can be observed by checking the validity of all the output bits.

To fight these remaining risks, three protections can be associated so as to increase the security level:

1) DPL, as detailed in the previous section.

2) Test for the existence of NULLs at the end of each computation. This sanity check basically consists in evaluating the Boolean security flag $\prod_{y \in\{\text { outputs }\}}\left(y_{t} \oplus y_{f}\right)[22]$.

3) Regular detection schemes, such as coding.

\section{Applicability of Resilience with CERTIFICATION Procedures}

The two main certification schemes of security products are the FIPS 140 and the common criteria. We examine in this section if the resilience can be applied with the current version of those standard, or if the standards are too conservative.

\footnotetext{
${ }^{7}$ Understand: as "close to perfect" as Boolean functions of finite dimensions can offer.
} 


\section{A. NIST FIPS 140-3}

The FIPS 140 [66], [67] formulates security requirements for cryptographic modules. It defines four levels of security, the highest of which is referred to as "security level 4". The functional security objectives of FIPS 140 are defined in $\S 3$. It includes those two requirements:

1) to detect errors in the operation of the cryptographic module and

2) to prevent the compromise or the modification of sensitive data and SSPs (Sensitive Security Parameters) resulting from these errors.

The "resilience" protection discussed in this article definitely fulfills the second requirement. However, not all resilient schemes comply with the first requirement. For instance, using the randomized homomorphic encryption (Algorithm 1), the errors cannot be detected. The partial resilience of dual-rail type countermeasure can allow a detection of the fault. However, the security of this scheme is ensured even if there is no detection. This means that FIPS-140 standards $2 \& 3$ are not resilienceready, although they express this idea.

More precisely, the exact statement of the requirements is detailed in $\S 4.5 .5$ (140-2 [66]) or $\S 4.6 .5$ (1403 [67]). For the security level 4, the cryptographic module shall either employ environmental failure protection (EFP) features or undergo environmental failure testing (EFT). The EFP consists in a constant monitoring of the environment (temperature and voltage) whereas EFP is an a priori characterization of the perturbation consequences. In both cases, the protection circuitry shall either (1) shutdown the module to prevent further operation or (2) immediately zeroize all plaintext secret and private cryptographic keys and SSPs.

Such authoritative and irremediable actions could have been prevented using a resilience scheme, without compromizing the device security. Therefore, we find that FIPS $140-\{2,3\}$ standards are too strict, resulting in potential inconveniences from the user perspective if non malicious faults cause the module shutdown or zeroization.

\section{B. Common Criteria}

The Common Criteria (CC) [1] is a framework that permits comparability between results of independent security evaluations. It is an international standard ISO/IEC 15408:2005. The CC in themselves do not specify security requirements. Instead, a "target of evaluation" (TOE) must meet "security targets" (ST). Zero, one or more "protection profiles" (PP) must be respected by the
ST. However, for marketing reasons, in practice, the ST complies to at least one PP. The security requirements are expressed in the PPs, whose structure is standardized but whose content is up to the designer. This flexibility allows a designer to tailor the PP to his (or that of his client) security objectives. Therefore, the $\mathrm{CC}$ readily accepts the resilience as a solution against fault attacks.

\section{Vi. Conclusions And Perspectives}

In embedded devices, fault attacks are usually combated in software. The dominant strategy is their detection, which is costly and non-exhaustive. We present in this paper an approach based on resilience. The faults are not necessarily captured, but the information they contain about any secret is nullified. The benefits of this approach are the ergonomy and the cost. First of all, the resilience impose no destruction of the secrets in case of a fault attack; thus, in case of natural (nonmalevolent faults) the user experience is a transient DoS, as opposed to a permanent DoS in traditional detectionbased countermeasures. Symmetrically, when a fault is injected successfully but has no consequence in the computation, a card protected with a detection-based scheme may react, whereas this inconvenience is nonexistent in the resilience-based scheme. Several concrete methods to implement resilient symmetrical encryption are proposed, amongst which a random mode of operation that is suitable for low-cost (without expensive module-level protections) smartcards. When the designer can propose a hardware counter-measure, we suggest the use of multi-valued or DPL styles. Those logics simultaneously protect against observation and perturbation attacks, and are cheaper than detection based on codes.

As a perspective, we intend to quantify the optimal parameters of code-based detection schemes that can be added to a DPL logic (evoked in Sec. IV-E) to further reduce the number of faulty results outputted by the device. Also, we thrive to define a formal framework based on the information theory that could describe with commensurable metrics the resistance of a cryptographic implementations to both SCA and FIA.

\section{ACKNOWLEDGMENTS}

The authors are very grateful to the five anonymous reviewers, that all contributed to improve the paper and to better place it in its scientific context. Novel ideas have also be suggested, that all open the door to efficient and formally proved countermeasures against active and passive attacks. We also thank the positive inputs received from the audience during the presentation at FDTC 
2010 in Santa Barbara, especially from Jean-Christophe Courrège, Guido Bertoni and Matthieu Rivain.

\section{REFERENCES}

[1] Common Criteria ( $a k a$ CC) for Information Technology Security Evaluation (ISO/IEC 15408).

Website: http://www.commoncriteriaportal.org/.

[2] Michel Agoyan, Jean-Max Dutertre, David Naccache, Bruno Robisson, and Assia Tria. When Clocks Fail: On Critical Paths and Clock Faults. In CARDIS, volume 6035 of Lecture Notes in Computer Science, pages 182-193. Springer, April 14-16 2010. Passau, Germany.

[3] Ross Anderson and Markus Kuhn. Tamper Resistance - a Cautionary Note. In In Proceedings of the Second USENIX Workshop ON Electronic Commerce, pages 1-11, 1996.

[4] Ross J. Anderson and Markus G. Kuhn. Low Cost Attacks on Tamper Resistant Devices. In Security Protocols Workshop, volume 1361 of Lecture Notes in Computer Science, pages 125136. Springer, April 7-9 1997. Paris, France.

[5] Yuichi Baba, Atsushi Miyamoto, Naofumi Homma, and Takafumi Aoki. Multiple-Valued Constant-Power Adder for Cryptographic Processors. In ISMVL, pages 239-244. IEEE Computer Society, May 21-23 2009. Naha, Okinawaw, Japan.

[6] Hagai Bar-El, Hamid Choukri, David Naccache, Michael Tunstal, and Claire Whelan. The Sorcerer's Apprentice Guide to Fault Attacks. Proceedings of the IEEE, 94(2):370-382, 2006. DOI: 10.1109/JPROC.2005.862424.

[7] Alessandro Barenghi, Guido Bertoni, Emanuele Parrinello, and Gerardo Pelosi. Low voltage fault attacks on the RSA cryptosystem. In FDTC, pages 23-31. IEEE Computer Society, September 6th 2009. Lausanne, Switzerland. DOI: 10.1109/FDTC.2009.30.

[8] Alessandro Barenghi, Guido Bertoni Luca Breveglieri, Mauro Pellicioli, and Gerardo Pelosi. Low Voltage Fault Attacks to AES. In HOST (Hardware Oriented Security and Trust). IEEE Computer Society, June 13-14 2010. Anaheim Convention Center, CA, USA.

[9] Friedrich Beck. Integrated Circuit Failure Analysis: A Guide to Preparation Techniques. Wiley, January 1998. ISBN-10: 0471974013; ISBN-13: 978-0471974017; 190 pages.

[10] Shivam Bhasin, Taoufik Chouta, Guillaume Duc, Jean-Luc Danger, Aziz El Aabid, Florent Flament, Philippe Hoogvorst, Tarik Graba, Sylvain Guilley, Houssem Maghr'ebi, Olivier Meynard, Maxime Nassar, Renaud Pacalet, Laurent Sauvage, Nidhal Selmane, and Youssef Souissi. Combined countermeasures against perturbation \& observation attacks. In PASTIS (PAca Security Trends In embedded Security), Gardanne (École des Mines de Saint-Étienne), France, June 16-17 2010. http://www.secure-ic.com/PDF/pastis_2010.pdf.

[11] Shivam Bhasin, Jean-Luc Danger, Florent Flament, Tarik Graba, Sylvain Guilley, Yves Mathieu, Maxime Nassar, Laurent Sauvage, and Nidhal Selmane. Combined SCA and DFA Countermeasures Integrable in a FPGA Design Flow. In ReConFig, pages 213-218. IEEE Computer Society, December 9-11 2009. Cancún, Quintana Roo, México, DOI: 10.1109/ReConFig.2009.50, http://hal.archives-ouvertes.fr/hal-00411843/en/.

[12] Shivam Bhasin, Sylvain Guilley, Florent Flament, Nidhal Selmane, and Jean-Luc Danger. Countering Early Evaluation: An Approach Towards Robust Dual-Rail Precharge Logic. In WESS. ACM, October 24-28 2010. Scottsdale, Arizona, USA. DOI: $10.1145 / 1873548.1873554$.
[13] Eli Biham and Adi Shamir. Differential Fault Analysis of Secret Key Cryptosystems. In CRYPTO, volume 1294 of LNCS, pages 513-525. Springer, August 1997. Santa Barbara, California, USA. DOI: $10.1007 / \mathrm{BFb} 0052259$.

[14] Dan Boneh, Richard A. DeMillo, and Richard J. Lipton. On the Importance of Checking Cryptographic Protocols for Faults. In Proceedings of Eurocrypt'97, volume 1233 of LNCS, pages 37-51. Springer, May 11-15 1997. Konstanz, Germany.

[15] Arnaud Boscher, Helena Handschuh, and Elena Trichina. Blinded Fault Resistant Exponentiation Revisited. In FDTC, pages 3-9. IEEE Computer Society, September 6 2009. Lausanne, Switzerland.

[16] Arnaud Boscher, Robert Naciri, and Emmanuel Prouff. CRT RSA Algorithm Protected Against Fault Attacks. In Information Security Theory and Practices. Smart Cards, Mobile and Ubiquitous Computing Systems, volume 4462 of LNCS, pages 229-243. Springer, May 9-11 2007. Heraklion, Crete, Greece.

[17] Suresh Chari, Charanjit S. Jutla, Josyula R. Rao, and Pankaj Rohatgi. Towards Sound Approaches to Counteract PowerAnalysis Attacks. In CRYPTO, volume 1666 of LNCS. Springer, August 15-19 1999. Santa Barbara, CA, USA. ISBN: 3-54066347-9.

[18] Zhimin Chen and Yujie Zhou. Dual-Rail Random Switching Logic: A Countermeasure to Reduce Side Channel Leakage. In CHES, volume 4249 of LNCS, pages 242254. Springer, October 10-13 2006. Yokohama, Japan, http://dx.doi.org/10.1007/11894063_20.

[19] Jean-Sébastien Coron and Avradip Mandal. PSS Is Secure against Random Fault Attacks. In ASIACRYPT, volume 5912 of LNCS, pages 653-666. Springer, December 6-10 2009. Tōkyō, Japan.

[20] Jean-Luc Danger and Sylvain Guilley. Circuit de cryptographie programmable - Logique BCDL (Balanced Cell-based Differential Logic), 25 Mars 2008. Brevet Français FR08/51904, assigné à l'Institut TELECOM; WO/2009/118264.

[21] Jean-Luc Danger, Sylvain Guilley, Shivam Bhasin, and Maxime Nassar. Overview of Dual Rail with Precharge Logic Styles to Thwart Implementation-Level Attacks on Hardware Cryptoprocessors, - New Attacks and Improved Counter-Measures -. In SCS, IEEE, pages 18, November 6-8 2009. Jerba, Tunisia. Complete version online: http://hal.archives-ouvertes.fr/hal-00431261/en/. DOI: 10.1109/ICSCS.2009.5412599.

[22] Jean-Luc Danger, Sylvain Guilley, and Florent Flament. Détection de faute dans un cryptoprocesseur protégé contre la DPA par logique différentielle, 12 Août 2008. Brevet Français FR08/55537, assigné à l'Institut TELECOM.

[23] Toshinori Fukunaga and Junko Takahashi. Practical fault attack on a cryptographic LSI with ISO/IEC 18033-3 block ciphers. In FDTC, pages 84-92. IEEE Computer Society, September 6th 2009. Lausanne, Switzerland. DOI: 10.1109/FDTC.2009.34.

[24] 3rd Generation Smart Card Project, G3Card; European project under grant IST-1999-13515. Website: http://www.g3card.org/.

[25] Berndt M. Gammel and Stefan Mangard. On the duality of probing and fault attacks. Cryptology ePrint Archive, Report 2009/352, 2009. http://eprint.iacr.org/.

[26] Rosario Gennaro, Anna Lysyanskaya, Tal Malkin, Silvio Micali, and Tal Rabin. Algorithmic Tamper-Proof (ATP) Security: Theoretical Foundations for Security against Hardware Tampering. In TCC, volume 2951 of Lecture Notes in Computer Science, pages 258-277. Springer, February 19-21 2004. Cambridge, MA, USA.

[27] Christophe Giraud and Hugues Thiebeauld. A Survey on Fault 
Attacks. In Kluwer, editor, Smart Card Research and Advanced Applications VI, IFIP 18th, World Computer Congress, TC8/WG8.8 \& TC11/WG11.2 Sixth International Conference on Smart Card Research and Advanced Applications (CARDIS), pages 159-176, 22-27 August 2004. Toulouse, France.

[28] Sudhakar Govindavajhala and Andrew W. Appel. Using Memory Errors to Attack a Virtual Machine. In SP'03: Proceedings of the 2003 IEEE Symposium on Security and Privacy, pages 154-165, Washington, DC, USA, May 11-14 2003. IEEE Computer Society. Berkeley, CA, USA.

[29] Jorge Guajardo and Bart Mennink. Towards Side-Channel Resistant Block Cipher Usage or Can We Encrypt Without SideChannel Countermeasures? Cryptology ePrint Archive, Report 2010/015, January 11 2010. http://eprint.iacr.org/2010/015.

[30] Sylvain Guilley, Sumanta Chaudhuri, Laurent Sauvage, JeanLuc Danger, Taha Beyrouthy, and Laurent Fesquet. Updates on the Potential of Clock-Less Logics to Strengthen Cryptographic Circuits against Side-Channel Attacks. In ICECS, IEEE, pages 351-354, December 13-16 2009. Medina, Yasmine Hammamet, Tunisia. DOI: 10.1109/ICECS.2009.5411008.

[31] Sylvain Guilley, Sumanta Chaudhuri, Laurent Sauvage, Tarik Graba, Jean-Luc Danger, Philippe Hoogvorst, Vinh-Nga Vong, and Maxime Nassar. Place-and-Route Impact on the Security of DPL Designs in FPGAs. In HOST, pages 29-35. IEEE Computer Society, June 9 2008. Anaheim, USA. ISBN $=978-$ 1-4244-2401-6.

[32] Sylvain Guilley, Sumanta Chaudhuri, Laurent Sauvage, Philippe Hoogvorst, Renaud Pacalet, and Guido Marco Bertoni. Security Evaluation of WDDL and SecLib Countermeasures against Power Attacks. IEEE Transactions on Computers, 57(11):14821497, nov 2008.

[33] Sylvain Guilley, Florent Flament, Renaud Pacalet, Philippe Hoogvorst, and Yves Mathieu. Security Evaluation of a Balanced Quasi-Delay Insensitive Library. In DCIS, Grenoble, France, nov 2008. IEEE. 6 pages, Session 5D - Reliable and Secure Architectures, ISBN: 978-2-84813-124-5, full text in HAL: http://hal.archives-ouvertes.fr/hal-00283405/en/.

[34] Sylvain Guilley, Philippe Hoogvorst, Yves Mathieu, and Renaud Pacalet. The "Backend Duplication" Method. In CHES, volume 3659 of LNCS, pages 383-397. Springer, 2005. August 29th September 1st, Edinburgh, Scotland, UK.

[35] Sylvain Guilley, Philippe Hoogvorst, Yves Mathieu, Renaud Pacalet, and Jean Provost. CMOS Structures Suitable for Secured Hardware. In DATE'04 - Volume 2, pages 1414-1415. IEEE Computer Society, February 2004. Paris, France. DOI: 10.1109/DATE.2004.1269113.

[36] Sylvain Guilley, Laurent Sauvage, Florent Flament, Philippe Hoogvorst, and Renaud Pacalet. Evaluation of PowerConstant Dual-Rail Logics Counter-Measures against DPA with Design-Time Security Metrics. IEEE Transactions on Computers, 9(59):1250-1263, September 2010. DOI: 10.1109/TC.2010.104.

[37] Helena Handschuh, Pascal Paillier, and Jacques Stern. Probing Attacks on Tamper-Resistant Devices. In CHES, volume 1717 of LNCS, pages 303-315. Springer, August 12-13 1999. Worcester, MA, USA.

[38] Yuval Ishai, Manoj Prabhakaran, Amit Sahai, and David Wagner. Private Circuits II: Keeping Secrets in Tamperable Circuits. In EUROCRYPT, volume 4004 of Lecture Notes in Computer Science, pages 308-327. Springer, May 28 - June 1 2006. St. Petersburg, Russia.

[39] Marc Joye and Michael Tunstall. Fault Analysis in Cryptography. Springer LNCS, March 2011. http://joye.site88.net/FAbook.html.

[40] Mark G. Karpovsky, Konrad J. Kulikowski, and Alexander Taubin. Robust Protection against Fault Injection Attacks on Smart Cards Implementing the Advanced Encryption Standard. In $D S N$, pages 93-101. IEEE Computer Society, June 28 - July 01 2004. Florence, Italy.

[41] Paul C. Kocher. Leak-resistant cryptographic indexed key update, March 25 2003. United States Patent 6,539,092 filed on July 2nd, 1999 at San Francisco, CA, USA.

[42] Paul C. Kocher, Joshua Jaffe, and Benjamin Jun. Differential Power Analysis. In Proceedings of CRYPTO'99, volume 1666 of LNCS, pages 388-397. Springer-Verlag, 1999.

[43] Oliver Kömmerling and Markus G. Kuhn. Design Principles for Tamper-Resistant Smartcard Processors. In WOST'99: Proceedings of the USENIX Workshop on Smartcard Technology on USENIX Workshop on Smartcard Technology, pages 2-2, Berkeley, CA, USA, 1999. USENIX Association. (On-line paper).

[44] Konrad J. Kulikowski, Mark G. Karpovsky, and Alexander Taubin. Power Attacks on Secure Hardware Based on Early Propagation of Data. In IOLTS, pages 131-138. IEEE Computer Society, 2006. Como, Italy.

[45] Régis Leveugle. Early Analysis of Fault-based Attack Effects in Secure Circuits. IEEE Trans. Computers, 56(10):1431-1434, 2007.

[46] Yang Li, Shigeto Gomisawa, Kazuo Sakiyama, and Kazuo Ohta. An Information Theoretic Perspective on the Differential Fault Analysis against AES. Cryptology ePrint Archive, Report 2010/032, 2010. http://eprint.iacr.org/.

[47] Yang Li, Kazuo Sakiyama, Shigeto Gomisawa, Toshinori Fukunaga, Junko Takahashi, and Kazuo Ohta. Fault Sensitivity Analysis. In CHES, volume 6225 of Lecture Notes in Computer Science, pages 320-334. Springer, August 17-20 2010. Santa Barbara, CA, USA.

[48] Lorentz Center, International Center for workshops in the Sciences. Workshop on "Provable Security against Physical Attacks", February 10-19 2010. Amsterdam, Netherlands. http://www.lorentzcenter.nl/lc/web/2010/383/program.php3?wsid=383.

[49] Vincent Maingot and Régis Leveugle. Influence of error detecting or correcting codes on the sensitivity to DPA of an AES S-box. In SCS, IEEE, pages 1-5, November 6-8 2009. Jerba, Tunisia. DOI: 10.1109/ICSCS.2009.5412600.

[50] Tal Malkin, François-Xavier Standaert, and Moti Yung. A Comparative Cost/Security Analysis of Fault Attack Countermeasures. In FDTC, volume 4236 of Lecture Notes in Computer Science, pages 159-172. Springer, October 10 2006. Yokohama, Japan.

[51] Stefan Mangard, Elisabeth Oswald, and Thomas Popp. Power Analysis Attacks: Revealing the Secrets of Smart Cards. Springer, December 2006. ISBN 0-387-30857-1, http://www.dpabook.org/.

[52] Robert P. McEvoy, Colin C. Murphy, William P. Marnane, and Michael Tunstall. Isolated WDDL: A Hiding Countermeasure for Differential Power Analysis on FPGAs. ACM Trans. Reconfigurable Technol. Syst. (TRETS), 2(1):1-23, 2009.

[53] Robert P. McEvoy, Michael Tunstall, Claire Whelan, Colin C. Murphy, and William P. Marnane. A differential side-channel analysis countermeasure. European Patent Application (EP 2148462 A1), filled in 27.01.2010.

[54] Robert P. McEvoy, Michael Tunstall, Claire Whelan, Colin C. Murphy, and William P. Marnane. All-or-Nothing Transforms as a Countermeasure to Differential Side-Channel Analysis. 
Cryptology ePrint Archive, Report 2009/185, April 302009. http://eprint.iacr.org/2009/185.

[55] Marcel Medwed, François-Xavier Standaert, Johann Großschädl, and Francesco Regazzoni. Fresh Re-Keying: Security against Side-Channel and Fault Attacks for Low-Cost Devices. In AFRICACRYPT, volume 6055 of $L N C S$, pages 279-296. Springer, May 03-06 2010. Stellenbosch, South Africa. DOI: 10.1007/978-3-642-12678-9_17.

[56] Yannick Monnet, Marc Renaudin, Régis Leveugle, Christophe Clavier, and Pascal Moitrel. Case Study of a Fault Attack on Asynchronous DES Crypto-Processors. In FDTC, volume 4236 of Lecture Notes in Computer Science, pages 88-97. Springer, October 10 2006. Yokohama, Japan.

[57] Simon Moore, Robert Mullins, Paul Cunningham, Ross Anderson, and George Taylor. Improving smart card security using self-timed circuits. In ASYNC (Asynchronous Circuits and Systems), pages 211- 218, April 2002. ISSN: 1522-8681, ISBN: 0-7695-1540-1j INSPEC Accession Number: 7321683.

[58] Simon W. Moore, Ross J. Anderson, Robert D. Mullins, George S. Taylor, and Jacques J. A. Fournier. Balanced self-checking asynchronous logic for smart card applications. Microprocessors and Microsystems, 27(9):421-430, 2003.

[59] Amir Moradi, Thomas Eisenbarth, Axel Poschmann, Carsten Rolfes, Christof Paar, Mohammad T. Manzuri Shalmani, and Mahmoud Salmasizadeh. Information Leakage of Flip-Flops in DPA-Resistant Logic Styles. Cryptology ePrint Archive, Report 2008/188, 2008. http://eprint.iacr.org/.

[60] Maxime Nassar, Shivam Bhasin, Jean-Luc Danger, Guillaume Duc, and Sylvain Guilley. BCDL: A high performance balanced DPL with global precharge and without early-evaluation. In DATE'10, pages 849-854. IEEE Computer Society, March 812 2010. Dresden, Germany.

[61] NIST/ITL/CSD. Data Encryption Standard. FIPS PUB 46-3, Oct 1999.

http://csrc.nist.gov/publications/fips/fips46-3/fips46-3.pdf.

[62] NIST/ITL/CSD. Advanced Encryption Standard (AES). FIPS PUB 197, Nov 2001.

http://csrc.nist.gov/publications/fips/fips197/fips-197.pdf.

[63] Pascal Paillier. Public-Key Cryptosystems Based on Composite Degree Residuosity Classes. In EUROCRYPT, volume 1592 of Lecture Notes in Computer Science, pages 223-238. Springer, May 2-6 1999. Prague, Czech Republic.

[64] Gilles Piret and Jean-Jacques Quisquater. A Differential Fault Attack Technique against SPN Structures, with Application to the AES and KHAZAD. In CHES, volume 2779 of $L N C S$, pages 77-88. Springer, September 2003. Cologne, Germany.

[65] Thomas Popp, Mario Kirschbaum, Thomas Zefferer, and Stefan Mangard. Evaluation of the Masked Logic Style MDPL on a Prototype Chip. In CHES, volume 4727 of LNCS, pages 81-94. Springer, Sept 2007. Vienna, Austria.

[66] NIST FIPS (Federal Information Processing Standards) publication 140-2. Security Requirements for Cryptographic Modules. page 69, May 252001.

[67] NIST FIPS (Federal Information Processing Standards) publication 140-3. Security Requirements for Cryptographic Modules (Draft, Revised). page 63, 09/11 2009.

[68] Robert Redelmeier. cpuburn, CPU testing utilities, June 16 2001. Software available on-line: http://pages.sbcglobal.net/redelm/ under GNU Public Licence.

[69] Francesco Regazzoni, Thomas Eisenbarth, Johann Großschädl, Luca Breveglieri, Paolo Ienne, Israel Koren, and Christof Paar. Power Attacks Resistance of Cryptographic S-Boxes with
Added Error Detection Circuits. In DFT, pages 508-516. IEEE Computer Society, September 26-28 2007. Rome, Italy.

[70] Ronald L. Rivest, Adi Shamir, and Leonard M. Adleman. A Method for Obtaining Digital Signatures and Public-Key Cryptosystems. Commun. ACM, 21(2):120-126, 1978.

[71] Bruno Robisson and Pascal Manet. Differential Behavioral Analysis. In CHES, volume 4727 of LNCS, pages 413-426. Springer, September 10-13 2007. Vienna, Austria.

[72] Laurent Sauvage, Sylvain Guilley, Jean-Luc Danger, Yves Mathieu, and Maxime Nassar. Successful Attack on an FPGAbased WDDL DES Cryptoprocessor Without Place and Route Constraints. In DATE, pages 640-645, Nice, France, apr 2009. IEEE Computer Society.

[73] Nidhal Selmane, Shivam Bhasin, Sylvain Guilley, Tarik Graba, and Jean-Luc Danger. WDDL is Protected Against Setup Time Violation Attacks. In FDTC, pages 73-83. IEEE Computer Society, September 6th 2009. In conjunction with CHES'09, Lausanne, Switzerland. DOI: 10.1109/FDTC.2009.40; Online version: http://hal.archives-ouvertes.fr/hal-00410135/en/.

[74] Nidhal Selmane, Sylvain Guilley, and Jean-Luc Danger. Setup Time Violation Attacks on AES. In EDCC, The seventh European Dependable Computing Conference, pages 91-96, Kaunas, Lithuania, may 7-9 2008. ISBN: 978-0-7695-3138-0, DOI: 10.1109/EDCC-7.2008.11.

[75] Rafael Soares, Ney Calazans, Victor Lomné, Philippe Maurine, Lionel Torres, and Michel Robert. Evaluating the robustness of secure triple track logic through prototyping. In SBCCI'08: Proceedings of the 21st annual symposium on Integrated circuits and system design, pages 193-198, New York, NY, USA, September 1-4 2008. ACM.

[76] Rafael Soares, Ney Calazans, Victor Lomne, Thomas Ordas, Philippe Maurine, Lionel Torres, and Michel Robert. Evaluation on FPGA of Triple Rail Logic Robustness against DPA and DEMA. In DATE, track A4 (Secure embedded implementations), pages 634-639. IEEE, April 20-24 2009. Nice, France.

[77] Danil Sokolov, Julian Murphy, Alexander Bystrov, and Alex Yakovlev. Design and Analysis of Dual-Rail Circuits for Security Applications. IEEE Trans. Comput., 54(4):449-460, 2005.

[78] François-Xavier Standaert, Tal Malkin, and Moti Yung. A Unified Framework for the Analysis of Side-Channel Key Recovery Attacks. In EUROCRYPT, volume 5479 of $L N C S$, pages 443-461. Springer, April 26-30 2009. Cologne, Germany.

[79] Daisuke Suzuki and Minoru Saeki. Security Evaluation of DPA Countermeasures Using Dual-Rail Pre-charge Logic Style. In CHES, volume 4249 of LNCS, pages 255269. Springer, October 10-13 2006. Yokohama, Japan. http://dx.doi.org/10.1007/11894063_21.

[80] The "Xilinx TMR Tool". Features description at this web page: http://www.xilinx.com/ise/optional_prod/tmrtool.htm.

[81] Kris Tiri, David Hwang, Alireza Hodjat, Bo-Cheng Lai, Shenglin Yang, Patrick Schaumont, and Ingrid Verbauwhede. A side-channel leakage free coprocessor IC in $0.18 \mu \mathrm{m}$ CMOS for Embedded AES-based Cryptographic and Biometric Processing. In $D A C$, pages 222-227. ACM, June 13-17 2005. San Diego, CA, USA.

[82] Kris Tiri and Ingrid Verbauwhede. A Logic Level Design Methodology for a Secure DPA Resistant ASIC or FPGA Implementation. In DATE'04, pages 246-251. IEEE Computer Society, February 2004. Paris, France. DOI: 10.1109/DATE.2004.1268856.

[83] Kris Tiri and Ingrid Verbauwhede. Place and Route for Secure 
Standard Cell Design. In Kluwer, editor, Proceedings of WCC / CARDIS, pages 143-158, Aug 2004. Toulouse, France.

[84] Michael Tunstall and Debdeep Mukhopadhyay. Differential Fault Analysis of the Advanced Encryption Standard using a Single Fault. Report 2009/575, 2009. http://eprint.iacr.org/2009/575.

[85] Olli Vertanen. Java Type Confusion and Fault Attacks. In FTDC, volume 4236 of LNCS, pages 237-251. Springer, 2006. DOI: $10.1007 / 11889700$, ISSN 0302-9743 (Print) 1611-3349 (Online), ISBN 978-3-540-46250-7.

[86] Sung-Ming Yen and Marc Joye. Checking Before Output May Not Be Enough Against Fault-Based Cryptanalysis. IEEE Trans. Computers, 49(9):967-970, 2000. DOI: $10.1109 / 12.869328$.

[87] Sung-Ming Yen, Seungjoo Kim, Seongan Lim, and Sang-Jae Moon. RSA Speedup with Chinese Remainder Theorem Immune against Hardware Fault Cryptanalysis. IEEE Trans. Computers, 52(4):461-472, 2003. DOI: 10.1109/TC.2003.1190587.

[88] Zhongchuan C. Yu, Stephen B. Furber, and Luis A. Plana. An Investigation into the Security of Self-Timed Circuits. In ASYNC, pages 206-215. IEEE Computer Society, May 12-16 2003. Vancouver, BC, Canada.

[89] Daheng Yue, Yan Sun, Minxuan Zhang, Shaoqing Li, and Yutong Dai. A Look-Up-Table Based Differential Logic to counteract DPA attacks. In ASICON, pages 855-858. IEEE Computer Society, October 20-23 2009. Changsha, Hunan, China. DOI: 10.1109/ASICON.2009.5351561. 